\title{
Genomics and young populations: Unlocking the door to preventative medicine
}

\author{
Angie Karlos and Dustin Hittel
}

\author{
University of Calgary
}

On a population level, rapidly rising obesity rates have been attributed to physical inactivity and the consumption of calorie dense foods. Obesity and its associated comorbidities, such as type 2 diabetes (T2D) and cardiovascular disease (CVD), decrease quality of life and result in an estimated 35 million premature deaths annually. ${ }^{1,2}$ Despite extensive efforts to understand the pathophysiology of obesity and develop efficacious prevention, treatment, and management strategies, obesity rates continue to increase. A major challenge to understanding the etiology of obesity is the significant inter-individual variation in both susceptibility to and consequences of this disease. However, the development of genome-wide association studies (GWAS) has provided a novel method that can be used to investigate and explain many inter-individual differences in chronic diseases.

GWAS involve conducting unbiased genome-wide scans of thousands of subjects to identify common variants, or single nucleotide polymorphisms (SNPs), associated with a trait or disease of interest. ${ }^{3}$ GWAS, which require no a priori knowledge of biochemical mechanisms and pathways, can therefore lead to the discovery of novel genetic variants, and provide new insight into the pathophysiology of chronic diseases and continuous traits. Despite the successes of these studies, a major criticism of GWAS are their small effect sizes, whereby only a small percentage of the variation of each trait is accounted for by the SNPs identified by this method. This is particularly the case when compared to estimates of the contribution of genetic factors to a trait from family studies, in which traits that are similar between siblings are considered to be predominantly genetic in nature. ${ }^{4}$ For example, while a recently published GWAS identified 95 loci that were significantly associated with variability in lipid concentrations, these loci accounted for only $\sim 25-30 \%$ of the genetic variance in each lipid trait (TG, TC, LDL and HDL). ${ }^{5}$ In contrast, these traits were estimated to have $\sim 50-70 \%$ variability in family studies. ${ }^{6}$ The disparity in the levels of variance detected by GWAS compared to the estimated genetic contribution to those traits from family studies is a major obstacle to the clinical utility of GWAS. Therefore, a great amount of emphasis has been placed on determining whether an imperfect understanding of allelic architecture and/or SNP interactions with the environment, other genes, or age cause this "missing heritability".

The accumulation of environmental exposures such as physical inactivity or poor diet may cause an increase in the variability of complex traits and diseases, making genetic associations more difficult to detect. ${ }^{4}$ It has therefore been hypothesized that investigating genetic associations in young, healthy populations will yield larger effect sizes than are observed in older, symptomatic populations. More importantly, different genes may be involved at different stages of disease pathogenesis, and the identification of those associated with early, developmental stages may provide valuable targets with the potential to predict future disease risk. In addition, interventions developed to target genes involved in the development of, rather than the perpetuation of disease could lead to primary prevention.

GWAS investigating polygenic contributions to CVD have consistently found three SNPs on chromosome 1p13, in close proximity to the SORT1 gene, to be significantly associated with LDL and risk of CVD. In these studies, the minor, or less frequent, alleles were associated with LDL concentrations $0.1-0.2 \mathrm{mmol} / \mathrm{L}$ lower than those of the major alleles, as well as with a $9-13 \%$ decreased risk of developing CVD. ${ }^{7,8,9}$ Interestingly, smaller validation studies have shown that the $1 p 13.3$ locus accounts for a much larger proportion of the variability in LDL in younger, asymptomatic subjects than in older, symptomatic subjects (2.5-4.1\% versus $1 \%$, respectively). ${ }^{10}$ The larger effect of this variant in younger populations is significant, as it provides evidence of a gene-age interaction that accounts for some 
of the missing heritability in the 1p13-LDL relationship.

The field of genomic medicine is in its infancy; however, it is growing quickly as technology advances to meet the increasing demand for more efficient and cost-effective sequencing. While GWAS have successfully identified a large number of loci associated with chronic diseases in older populations, the evaluation of these associations in younger populations may provide better insight into disease etiology, as well as discover potentially valuable targets for disease prevention. Genetic risk stratification in children may prove to be a more valuable screening method for disease risk than measures such as insulin for T2D and LDL for CVD. Furthermore, in diseases that do not have an intermediate measure, such as obesity, genotype has the potential to be an extremely valuable indicator of disease risk that could be used early in life for disease prevention. Identifying genetic loci associated with the development of disease pathophysiology at a young age, as well as elucidating the mechanisms underlying these pathologies, will result in advancements in the prevention and treatment of complex diseases such as T2D and CVD.

\section{References}

1. Lustig, R.H., L.A. Schmidt, and C.D. Brindis, Public health: The toxic truth about sugar. Nature, 2012. 482(7383): p. 27-29.

2. Manson, J.E., et al., The escalating pandemics of obesity and sedentary lifestyle. A call to action for clinicians. Archives of Internal Medicine, 2004. 164(3): p. 249-258.

3. Wang, Y., et al., Bioinformatics and Public Access Resources. Genetic and Molecular Aspects of Sport Performance, 2011: p. 58-69.

4. Dumitrescu, L., et al., Evidence for Age As a Modifier of Genetic Associations for Lipid Levels. Annals of Human Genetics, 2011. 75: p. 589-597.

5. Teslovich, T.M., et al., Biological, clinical and population relevance of 95 loci for blood lipids. Nature, 2010. 466(7307): p. 707-713.

6. Perusse, L., et al., Familial resemblance of plasma lipids, lipoproteins and postheparin lipoprotein and hepatic lipases in the HERITAGE Family Study. Arterioscler Thromb Vasc Biol, 1997. 17(11): p. 3263-9.

7. Musunuru, K., et al., From noncoding variant to phenotype via SORT1 at the 1 p13 cholesterol locus. Nature, 2010. 466(7307): p. 714-719.

8. Linsel-Nitschke, P., et al., Genetic variation at chromosome 1p13. 3 affects sortilin mRNA expression, cellular LDL-uptake and serum LDL levels which translates to the risk of coronary artery disease. Atherosclerosis, 2010. 208(1): p. 183-189.

9. Kathiresan, S., et al., Common variants at 30 loci contribute to polygenic dyslipidemia. Nature genetics, 2008. 41(1): p. 56-65.

10. Devaney, J.M., et al., The 1p13. 3 LDL (C)-Associated Locus Shows Large Effect Sizes in Young Populations. Pediatric Research, 2011. 69(6): p. 538543.

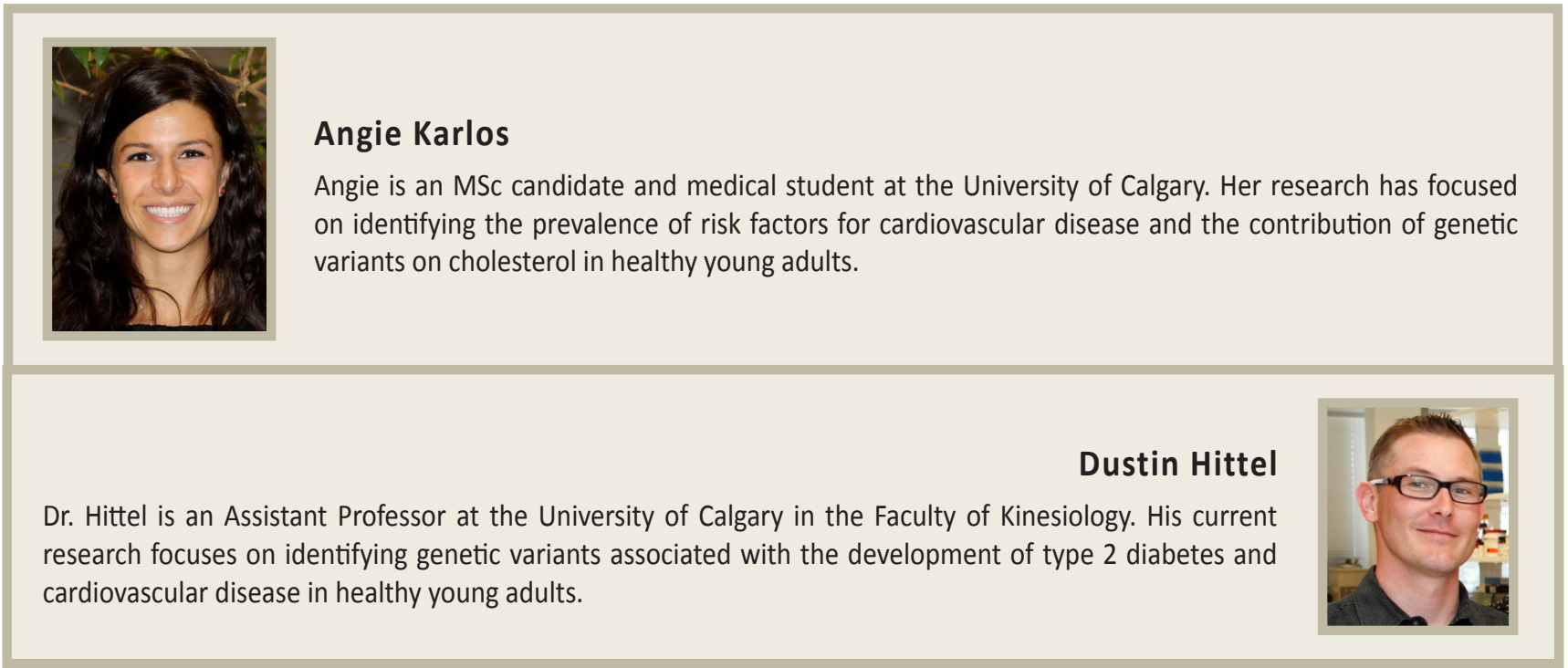

